\title{
Markov-chain Based Lottery Analysis System in Mobile Cloud Computing
}

\author{
He Yi ${ }^{1, a}$, Fu Rong ${ }^{1, b}$, Zhang Yingqian ${ }^{1, c}$, Wu Shuai ${ }^{1}$, Liu Xin ${ }^{1}$, Yuan Tian ${ }^{1}$ \\ City Institute, Dalian University of Technology, Dalian, Liaoning Province, 116600, P. R. China \\ a heyi517@126.com , b furong880@126.com, ' zhangyq@dlut.edu.cn
}

Keywords: mobile cloud computing; Markov chain; lottery analysis and forecasting; transition matrix

\begin{abstract}
This paper makes use of Markov chain to establish predictive model and analyze the historical data of lottery based on technologies of mobile cloud computing and HTML5 in order to predict the number sets with high-winning probability during next issue. It is indicated in the actual application that the system is capable of offering lottery buyers the analysis and forecasting service of lottery information anytime and anywhere, while effectively narrowing the scope of lottery winning with some practical significance.
\end{abstract}

\section{Introduction}

Thanks to the rapid development of mobile Internet, the wireless Internet access via mobile phones becomes quick and convenient. As of June 2015, there are 594 million Internet users via mobile phones in China, thus there are growing services of cloud computing of different kinds based on mobile terminal like mobile phones [1]. Meanwhile China has become a leading and big country of lottery sales in the world, and the future of lottery industry is revolves around the analysis of lottery big data and other aspects [2]. There are a large number of analysis and forecasting methods or computational formula of lottery historical data at present. In this paper, based on the mobile cloud computing technology, the Markov chain algorithm is employed to establish models, design and achieve the cross-platform lottery analysis system via smart phones. The system provide a set of lottery numbers with high winning rate, and dedicated to the analysis by numerous lottery buyers, so ad to effective enhancement of lottery winning rate.

\section{Markov chain}

Markov chain is an analysis method of random events sequence, and also used to forecast the state and development trend of incidents based on the transition probability matrix between states [3]. It is supposed that $\left\{X_{n}, n \in S\right\}, S=\{0,1,2,3 \ldots\}$ is a stochastic process featured by discrete state space and non-negative parameters, then $\left\{X_{(n)}\right\}$ meets following conditions:

$$
P\left\{X_{(n+1)}=j \mid X_{(0)}=i_{0}, X_{(1)}=i_{1}, \ldots, X_{(n-1)}=i_{n-1}, X_{(n)}=i_{n}\right\}=P\left\{X\left(_{n+1)}=j \mid X_{(n)}=i\right\}\right.
$$

The transition probability matrix is derived through based on the statistics of frequency $f_{(\mathrm{i}, \mathrm{j})}$ historical data. $f_{(\mathrm{i}, \mathrm{j})}$ represents the transitioning times from the state $i$ to state $j$; then the probability transfer matrix

$$
P=\left\{p_{i j}, i, j \in S\right\}=\left[\begin{array}{ccc}
p_{11} & \ldots & p_{1 n} \\
\ldots & \ldots & \ldots \\
p_{m 1} & \ldots & p_{m n}
\end{array}\right]
$$

It is called one-step transition probability matrix of Markov chain $\left\{X_{n}, n \in S\right\}$, with features as below: $p_{i j} \geq 0(i, j \in S)$ and $\sum_{j \in S}=1(i \in S)$.

The m-step transition probability matrix can also be defined. The matrix with features of (1) and (2) is called m-step transition matrix. 


\section{C-K Equation}

Chapman -Kolmogorov equation is abbreviated as C-K equation, which plays an important role in the calculation of transition probability of Markov chain. Its formula is:

$$
p_{i j}^{(m+r)}(n) \sum_{k \in S} p_{i k}^{(m)}(n) p_{k j}^{(r)}(m+n), i, j \in S \text {. }
$$

This equation shows that the process starts at the state $i$, and then shifts to the state $j$ after $(m+r)$ steps. During the process it takes $m$ steps for the state $i$ to be shifted to the intermediate state $k$, which is then shifted to state $j$ through remaining $r$ steps.

\section{Establishment of mathematical model}

The mathematical model may be established regarding a variety of historical data of lottery winning numbers based on Markov chain for the prediction of winning numbers of next issue. In this paper, taking double chromosphere as an example, a mathematical model is established. The states of double chromosphere of 003 issue in 2015 is predicted based on the historical data of double chromosphere from 05 issue in 2014 to 002 issue in 2015(including 150 issues).

Taking the winning blue number of double chromosphere as an example, the selection of blue number is 1 out of 16 . Therefore each two consecutive datum are set as one state to obtain 8 states of Markov chain, divided into sets of states $S=\{1,2,3,4,5,6,7,8\}$. The specific state division is as shown in Table 1.

Table.1 State division of blue number

\begin{tabular}{ccccccccccc}
\hline $\begin{array}{c}\text { Blue } \\
\text { number }\end{array}$ & 1,2 & 3,4 & 5,6 & 7,8 & 9,10 & 11,12 & 13,14 & 15,16 \\
\hline State & 1 & 2 & 3 & 4 & 5 & 6 & 7 & 8 \\
\hline
\end{tabular}

The history of the lottery data state about blue number is derived from Table 1, with the historical state of winning blue number from 05 issue in 2014 to 002 issue in 2015[4].

When one-step transition probability is calculated, the frequencies can be obtained based on numbers of frequencies appeared at each state transition, thus the transition probability matrix is derived from formula (1) - (3). For example when efforts are made to get the value of $p_{11}$, there are 17 original samples in state 1 can be seen, and 2 samples shifting from state 1 to state 1 (respectively the issue of 2014108 and 2014134), so $p_{11}=2 / 17$. With the same method, one-step transition frequency matrix $\left(f_{i, j}\right)_{8 \times 8}$ and one-step probability matrix $\left(p_{i, j}\right)_{8 \times 8}$ of blue number state from issue 05 in 2014 to issue 002 in 2015 from Table 2 can be obtained through statistics:

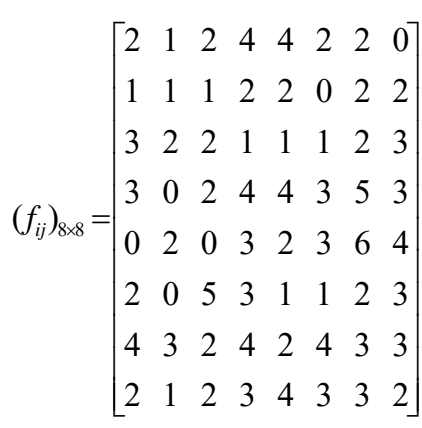

$$
\left(p_{i j}\right)_{8 \times 8}=\left[\begin{array}{cccccccc}
0.117 & 0.058 & 0.117 & 0.235 & 0.235 & 0.117 & 0.117 & 0 \\
0.090 & 0.090 & 0.090 & 0.181 & 0.181 & 0 & 0.181 & 0.181 \\
0.2 & 0.133 & 0.133 & 0.066 & 0.066 & 0.066 & 0.133 & 0.2 \\
0.125 & 0 & 0.083 & 0.166 & 0.166 & 0.125 & 0.208 & 0.125 \\
0 & 0.1 & 0 & 0.15 & 0 & 0.15 & 0.3 & 0.2 \\
0.117 & 0 & 0.294 & 0.176 & 0.058 & 0.058 & 0.117 & 0.176 \\
0.16 & 0.12 & 0.08 & 0.16 & 0.08 & 0.16 & 0.12 & 0.12 \\
0.1 & 0.05 & 0.1 & 0.15 & 0.2 & 0.15 & 0.15 & 0.1
\end{array}\right]
$$

Similarly, the state division of red number of double chromosphere is like blue number. The historical lottery-winning data states of red number can be obtained according to the historical lottery-winning data about red number of double chromosphere, thus one-step frequency and one-step transition probability matrix of red number state sequence are derived through statistics. 


\section{Model-based analysis and forecasting}

Probabilities of blue numbers in each state at the known moment $l$ are considered the initial distribution of Markov chain:

$X^{(0)}=\left(x_{1}^{(0)}, x_{2}^{(0)}, \ldots, x_{8}^{(0)}\right)$

According to the forecasting model of Markov chain, is the state transition matrix, thus the absolute probability distribution of each state at the time of $l+1$ is obtained.

$X^{(1)}=\left(x_{1}^{(1)}, x_{2}^{(1)}, \ldots, x_{8}^{(1)}\right)$

Thus the state $X^{(k)}=\left(x_{1}^{(k)}, x_{2}^{(k)}, \ldots, x_{8}^{(k)}\right)$ at the moment of $l+k$ is anticipated, then when the state $j$ satisfies $x_{j}^{(k)}=\max \left\{x_{j}^{(k)}\right\} \quad$ (in which $1 \leq j \leq 8$ ) the due states of blue number are predicted.

For example the blue number state of issue 2015002 is 3 , and it can further be seen from the one-step transfer matrix that the probability for the transition from state 3 to state 1 and 8 is the greatest. For more accurate prediction, the Markov chain forecasting can be superimposed, namely adding the corresponding figures of one-step probability matrix $p(1)$ and two-step Markov matrix $p$ (2), then the winning numbers of previous issue is further anticipated. For example, the sum of one-step transition probability of state 3 and two-step transition probability is shown in Table 2 as below.

Table 2 Sum of one-step transition probability and two-step transition probability

\begin{tabular}{lcccccccc}
\hline $\begin{array}{l}\text { one-step } \\
\text { transition } \\
\text { probability }\end{array}$ & 0.2 & 0.133 & 0.133 & 0.066 & 0.066 & 0.066 & 0.133 & 0.2 \\
\hline $\begin{array}{l}\text { two-step } \\
\text { transition } \\
\text { probability }\end{array}$ & 0.110 & 0.068 & 0.101 & 0.153 & 0.142 & 0.098 & 0.142 & 0.111 \\
\hline \multicolumn{1}{c}{ Sum } & 0.310 & 0.201 & 0.234 & 0.219 & 0.208 & 0.164 & 0.275 & 0.311 \\
\hline
\end{tabular}

As it can be seen from Table 2,state 8 has the greatest probability and the blue number of issue $2,015,003$ is actually in state 8 (blue number: 15). The prediction is accurate. In addition, the state of red number of issue 2015002 is $\{3,5,6,9,10,11\}$. It can be seen from the transition matrix that the set with maximum probability in the next state is $\{4,6,7,10,11,1\}$. To improve accuracy, the two-step transition probability of red number is also calculated and the adding it with one-step transition probability for summing. The red-number state of issue 2015003 is finally anticipated as $\{4,6,7,11,1\}$ while the red number of issue 201500 is in the state set of $\{4,5,7,8,11\}$. Thus the state of $4,7,11$ is accurately predicted.

\section{System implementation}

Currently the storage capacity and computing power of smart phone system are so limited, that a large amounts of data storage, processing and complex calculations in the system are completed by cloud server [5]. The system architecture is shown in Figure 1.

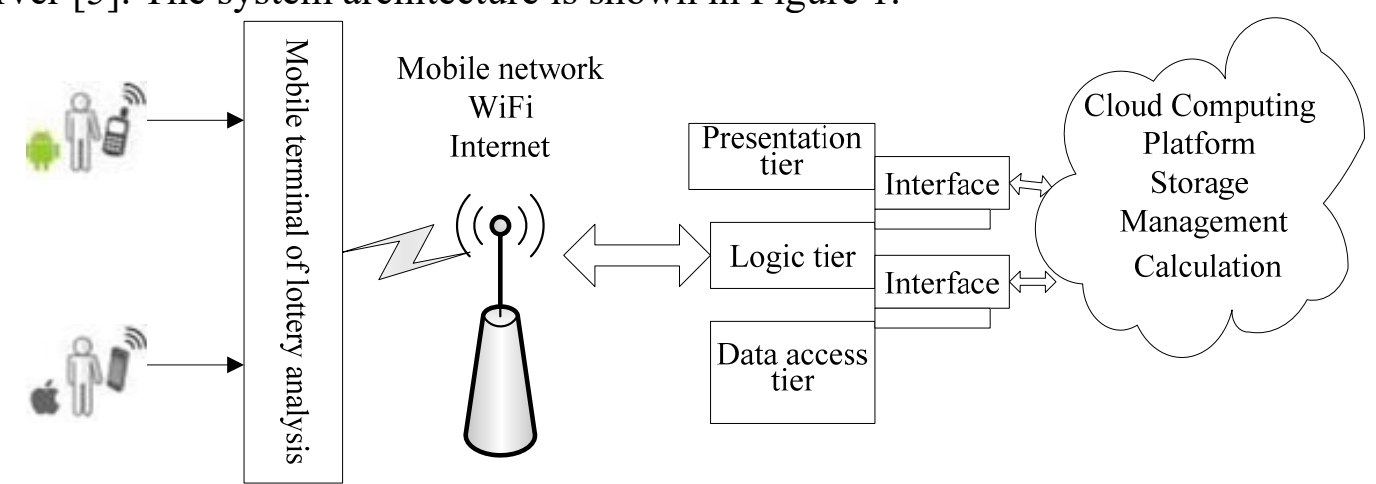

Fig.1 Service Model of System

The application makes use of cloud service technology of Alibaba to construct cloud 
resources of lottery analysis system, completing numerous calculation of data analysis through the cloud processing. Meanwhile the required tremendous historical data is also stored in "cloud". The mobile terminal platforms targeted by the system are Android and iOS. Therefore with the application of HTML5 cross-platform technological support, improving the development efficiency and reducing the development effort.

\section{Conclusion}

The cross-platform system of lottery analysis is designed and realized using Markov model based on the mobile cloud computing technology targeted at the strong randomness of lottery data. From the model result, the transition probability matrix can be used to deeply reflect the internal relations of dynamic changing mechanism of lottery, so as to provide sets of next issue with high-probability winning. In addition the system offers lottery buyers convenient and rapid services like analysis and forecasting, lottery inquiry, trend charts and earnings calculation to improve the lottery winning rate.

\section{Reference}

[1]LIU Feng. Analysis of the current situation and development trend of mobile Internet[J]. Electronic Technology \& Software Engineering. 2016,1(2):16-17

[2]ZHONG Jiang, JIANG Xiao Xia, LI Yin Yin. Analysis on the present situation and Countermeasures of sports lottery market in China $[\mathrm{J}]$. Contemporary Sports Technology. 2015,5(19):147-149.

[3]HUANG Gang. Applications of Markov model and hidden Markov model[J]. Electronic Design Engineering. 2013, 21(17):60-62.

[4] http: //baidu.lecai.com

[5]SUN Yaoyao. Research on Cloud Computing Application on Mobile Internet[J]. Electronic Sci \& Tech. 2015,28(1):179-182. 